\title{
A component-by-component construction for the trigonometric degree
}

Nico Achtsis and Dirk Nuyens

— In memory of James Lyness (1932-2010) -

\begin{abstract}
We propose an alternative to the algorithm from Cools, Kuo, Nuyens (Computing, 2010) [5], for constructing lattice rules with good trigonometric degree. The original algorithm has construction cost $O\left(\left|\mathscr{A}_{d}(m)\right|+d N \log N\right)$ for an $N$-point lattice rule in $d$ dimensions having trigonometric degree $m$, where the set $\mathscr{A}_{d}(m)$ has exponential size in both $d$ and $m$ (in the "unweighted degree" case, which is what we consider here). We reduce the cost to $O\left(d N(\log N)^{2}\right)$ with an implicit constant governing the needed precision (which is dependent on $N$ and $d$ ).
\end{abstract}

\section{Introduction}

Consider $d$-dimensional integrand functions $f$ having absolutely convergent Fourier series representation

$$
f(\boldsymbol{x})=\sum_{\boldsymbol{h} \in \mathbb{Z}^{d}} \hat{f}(\boldsymbol{h}) \mathrm{e}^{2 \pi \mathrm{i} h \cdot \boldsymbol{x}}
$$

then the error of integration by means of a rank-1 lattice rule $[19,24]$ is given by

$$
Q(f ; \boldsymbol{z}, N)-I(f)=\frac{1}{N} \sum_{k=0}^{N-1} f\left(\frac{k \boldsymbol{z} \bmod N}{N}\right)-\int_{[0,1)^{d}} f(\boldsymbol{x}) \mathrm{d} \boldsymbol{x}=\sum_{\substack{\boldsymbol{h} \in \mathbb{Z}^{d} \backslash\{\mathbf{0}\} \\ \boldsymbol{h} \cdot \boldsymbol{z} \equiv 0 \quad(\bmod N)}} \hat{f}(\boldsymbol{h}),
$$

Nico Achtsis, Dirk Nuyens

Department of Computer Science, K.U.Leuven, Celestijnenlaan 200A, 3001 Heverlee, Belgium, e-mail: nico.achtsis@cs.kuleuven.be, e-mail: dirk.nuyens@cs.kuleuven.be 
where $I(f)$ is the integral of $f$ and $Q(f ; z, N)$ its approximation by an $N$-point (rank-1) lattice rule with integer generating vector $\boldsymbol{z}$. The set $\Lambda^{\perp}:=\left\{\boldsymbol{h} \in \mathbb{Z}^{d}\right.$ : $\boldsymbol{h} \cdot \boldsymbol{z} \equiv 0(\bmod N)\}$, appearing in (1), is called the dual lattice (for the lattice $\Lambda$ with generator $\boldsymbol{z} / N+\mathbb{Z}^{d}$ ). We want to construct lattice rules which integrate exactly all Fourier coefficients which are at most a distance $m$ from the origin measured by the 1-norm. The largest such $m$, for a fixed rule $Q$, then denotes the trigonometric degree of the lattice rule. Figure 1 shows the Fourier space for the trigonometric degree, as well as for the product trigonometric degree, which measures the distance in the $\infty$-norm, to be used in the next section. The trigonometric degree and similar quantities, originating in the Russian literature, have been studied in many Western publications, some of them by James Lyness $[6,16,17,18]$; other references are $[1,11,10,7,3,2,8,9,5,23]$.

One is able to easily write down the reproducing kernel of such a (finite) dimensional reproducing kernel Hilbert space (RKHS) in terms of an orthonormal basis. For a space of functions of trigonometric degree at most $m$ we get

$$
K_{m}(\boldsymbol{x}, \boldsymbol{y})=\sum_{\substack{\boldsymbol{h} \in \mathbb{Z}^{d} \\\|\boldsymbol{h}\|_{1} \leq m}} \exp (2 \pi \mathrm{i} \boldsymbol{h} \cdot \boldsymbol{x}) \overline{\exp (2 \pi \mathrm{i} \boldsymbol{h} \cdot \boldsymbol{y})}=\sum_{\substack{\boldsymbol{h} \in \mathbb{Z}^{d} \\\|\boldsymbol{h}\|_{1} \leq m}} \exp (2 \pi \mathrm{i} \boldsymbol{h} \cdot(\boldsymbol{x}-\boldsymbol{y})) .
$$

The squared worst-case error using a rank-1 lattice rule in this RKHS is then given by

$$
e^{2}\left(\boldsymbol{z}, N ; K_{m}\right)=-1+\frac{1}{N} \sum_{k=0}^{N-1} \sum_{\substack{\boldsymbol{h} \in \mathbb{Z}^{d} \\\|\boldsymbol{h}\|_{1} \leq m}} \exp (2 \pi \mathrm{i} \boldsymbol{h} \cdot(k \boldsymbol{z}) / N),
$$

see, e.g., [15] for expressing worst-case errors in a RKHS. The worst-case error for a quadrature/cubature rule $Q$ in a Banach space $\mathscr{H}$ is defined as

$$
e(Q ; \mathscr{H}):=\sup _{\substack{f \in \mathscr{H} \\\|f\|_{\mathscr{H}} \leq 1}}|I(f)-Q(f)| .
$$

If the rank-1 rule specified by $z$ and $N$ has trigonometric degree $m$, then its worstcase error in the RKHS with kernel $K_{m}$ will be zero. The latter form for the squared worst-case error (3) is, for $d \gg 1$, far from convenient for construction purposes as the sum over the Fourier indices $\boldsymbol{h}$ cannot be written in a "product form". A kernel which can be written in a product form (or a small sum of product forms) is a necessary condition for the current fast component-by-component algorithms, see, e.g., [21] and [4] for some example kernels.

For comparison, the classical infinite dimensional function space which takes all Fourier coefficients into account, the so-called Korobov space, has reproducing kernel, for $\alpha>1$,

$$
K_{\alpha}(\boldsymbol{x}, \boldsymbol{y})=\sum_{\boldsymbol{h} \in \mathbb{Z}^{d}} \frac{\exp (2 \pi \mathrm{i} \boldsymbol{h} \cdot(\boldsymbol{x}-\boldsymbol{y}))}{\prod_{j=1}^{d} \max \left(1,\left|h_{j}\right|^{\alpha}\right)}=\prod_{j=1}^{d}\left(1+\sum_{0 \neq h \in \mathbb{Z}} \frac{\exp \left(2 \pi \mathrm{i} h\left(x_{j}-y_{j}\right)\right)}{|h|^{\alpha}}\right),
$$


where the infinite sum reduces to a Bernoulli polynomial $B_{\alpha}$ in case $\alpha$ is even. The squared worst-case error using a rank-1 lattice rule is then

$$
e^{2}\left(z, N ; K_{\alpha}\right)=-1+\frac{1}{N} \sum_{k=0}^{N-1} \prod_{j=1}^{d}\left(1+c_{\alpha} B_{\alpha}\left(\frac{k z \bmod N}{N}\right)\right),
$$

for some easily determined constant $c_{\alpha}$.

The kernels we consider here are all in terms of Fourier series, therefore they are what is called shift-invariant or periodic, i.e., $K(\boldsymbol{x}, \boldsymbol{y})=K(\boldsymbol{x}-\boldsymbol{y}, \mathbf{0})$. In general the squared worst-case error for a shift-invariant space with kernel $K$ using a lattice rule is given by

$$
e^{2}(\boldsymbol{z}, N ; K)=-\int_{[0,1)^{d}} K(\boldsymbol{x}, \mathbf{0}) \mathrm{d} \boldsymbol{x}+\frac{1}{N} \sum_{k=0}^{N-1} K(\boldsymbol{z} k / N, \mathbf{0}) .
$$

Using the Fourier expansion of $K(\boldsymbol{x}, \mathbf{0})=K_{\mathbf{0}}(\boldsymbol{x})$, i.e., the kernel with one leg fixed, we arrive at

$$
e^{2}(\boldsymbol{z}, N ; K)=-\widehat{K}_{\mathbf{0}}(\mathbf{0})+\sum_{\boldsymbol{h} \in \mathbb{Z}^{d}} \widehat{K}_{\mathbf{0}}(\boldsymbol{h}) \frac{1}{N} \sum_{k=0}^{N-1} \exp (2 \pi \mathrm{i} \boldsymbol{h} \cdot \boldsymbol{z} k / N)=\sum_{\substack{\mathbf{0} \neq \boldsymbol{h} \in \mathbb{Z}^{d} \\ \boldsymbol{h} \cdot \boldsymbol{z} \equiv 0}} \widehat{K}_{\mathbf{0}}(\boldsymbol{h}),
$$

where the latter sum is over the dual lattice. If one compares with (1) then it is clear that (5) is the integration error of the function $K_{0}(\boldsymbol{x})$ using the lattice rule $Q(\cdot ; z, N)$. In other words: the squared worst-case error of a lattice rule (in a shift-invariant space) is given as the sum of the Fourier coefficients of the kernel (with one leg fixed to $\mathbf{0}$ ) over the dual lattice. Therefore, the Fourier coefficients attach a weight to the dual lattice points in the squared worst-case error; this will be the point of view we will use in the following.

\section{Embedding by a tensor product function space}

In [5] a new algorithm was introduced to construct rank-1 lattice rules using a component-by-component procedure that obtains a prescribed weighted degree of exactness and, at the same time, achieve the near optimal worst-case error in a Korobov space. (The algorithm in [5] is presented for $N$ prime, but can be modified for composite $N$ as well. Also, the algorithm there is presented for different kinds of degrees of exactness, here we are only concerned with the trigonometric degree.) This algorithm explicitly constructs a set of Fourier indices $\mathscr{A}_{d}(m)$ associated with the degree of exactness, i.e., all integer points at a distance smaller than or equal to $m$ to the origin. The construction cost of that algorithm is $O\left(\left|\mathscr{A}_{d}(m)\right|+d N \log N\right)$. To make this algorithm feasible the degree of exactness is weighted by weights $\beta_{j}$ w.r.t. the different coordinate axes $j=1, \ldots, d$. If all these weights are put equal to 1 then one 
obtains the classical trigonometric degree and the size of the set $\mathscr{A}_{d}(m)$ increases exponentially in $d$ and $m$, making the construction intractable. More precisely, it can be shown, see, e.g., [11], that

$$
\left|\mathscr{A}_{d}(m)\right|=\left|\mathscr{A}_{m}(d)\right|=\sum_{s \geq 0} 2^{s}\left(\begin{array}{c}
d \\
s
\end{array}\right)\left(\begin{array}{c}
m \\
s
\end{array}\right) \leq \begin{cases}(1+2 m)^{d}=O\left((2 m)^{d}\right), & \text { if } d \leq m, \\
(1+2 d)^{m}=O\left((2 d)^{m}\right), & \text { if } m \leq d,\end{cases}
$$

where we used the Binomial theorem and the easy estimate $\left(\begin{array}{l}n \\ k\end{array}\right) \leq n^{k} / k ! \leq n^{k}$. (Note that the sum in (6) always has a finite summation range as both $d$ and $m$ are finite positive integers and $\left(\begin{array}{l}n \\ k\end{array}\right)=0$ for $k \notin\{0, \ldots, n\}, k, n \in \mathbb{Z}, n \geq 0$.)

In [5] the theoretical basis starts off by modifying the classical Korobov space to incorporate the kernel of the finite dimensional space, which is (2) for the trigonometric degree. The unfortunate form of this kernel plays no part there as one constructs the set $\mathscr{A}_{d}(m)$ explicitly and thus no calculations have to be done using this kernel. Here we propose to walk the other way: we will not build the (exponentially growing) set $\mathscr{A}_{d}(m)$, but will try to calculate the worst-case error for a modified trigonometric space.

Incorporating an idea from [13] we build a function space with exponentially decaying Fourier coefficients, and, extending what is studied in [13], make it finite dimensional. Our first attempt at an efficient kernel is

$$
K_{m, p}(\boldsymbol{x}, \boldsymbol{y})=\sum_{\substack{\boldsymbol{h} \in \mathbb{Z}^{d} \\\|\boldsymbol{h}\|_{1} \leq m}} p^{\|\boldsymbol{h}\|_{1}} \exp (2 \pi \mathrm{i} \boldsymbol{h} \cdot(\boldsymbol{x}-\boldsymbol{y})),
$$

for $0<p<1$. Note that the part inside the sum is now of "product form", however the multiple sums are a dependent chain. If one strives for exactness, i.e., integrate all these Fourier coefficients exactly, then there is no difference in using kernel (2) or (7). A rule which is exact for all trigonometric polynomials up to degree $m$ will have a squared worst-case error equal to zero for both of these choices. Moreover, as the sum still involves the 1-norm, we still fail to have an efficient computable form.

Now we enlarge the index set of Fourier coefficients to take a tensor product form. Again for $0<p<1$, now consider the kernel

$$
\begin{aligned}
K_{m, p}^{\prime}(\boldsymbol{x}, \boldsymbol{y}) & =\sum_{\substack{\boldsymbol{h} \in \mathbb{Z}^{d} \\
\|\boldsymbol{h}\|_{\infty} \leq m}} p^{\|\boldsymbol{h}\|_{1}} \exp (2 \pi \mathrm{i} \boldsymbol{h} \cdot(\boldsymbol{x}-\boldsymbol{y})) \\
& =\prod_{j=1}^{d} \sum_{h=-m}^{m} p^{|h|} \exp \left(2 \pi \mathrm{i} h\left(x_{j}-y_{j}\right)\right) \\
& =\prod_{j=1}^{d}\left(1+2 \sum_{h=1}^{m} p^{h} \cos \left(2 \pi h\left(x_{j}-y_{j}\right)\right)\right) .
\end{aligned}
$$

The last form is suitable to be directly used in the fast component-by-component algorithm [20, 22, 21]. 
The problem with (8) however is that we are now in fact looking at a product trigonometric degree (i.e., a tensor product form degree) instead of the plain trigonometric degree: that is, if the squared worst-case error for this kernel is zero, then the rule has product trigonometric degree at least $m$ (and by extension also trigonometric degree at least $m$ ), if it is non-zero however, then we could still have trigonometric degree at least $m$. This simple embedding can be seen in Figure 1. We want to obtain bounds on the value for the squared worst-case error such that we can determine, when it is non-zero, if the Fourier coefficients for $\|\boldsymbol{h}\|_{\infty} \leq m$, which we don't integrate exactly (i.e., the dual lattice points), actually have $\|\boldsymbol{h}\|_{1}>m$, i.e., all in the shaded area in Figure 1 (but not on the border of the inner diamond). If so, then the rule has trigonometric degree at least $m$ (right image; with actual trigonometric degree $m$ ), if not, then the rule has smaller degree (left image).
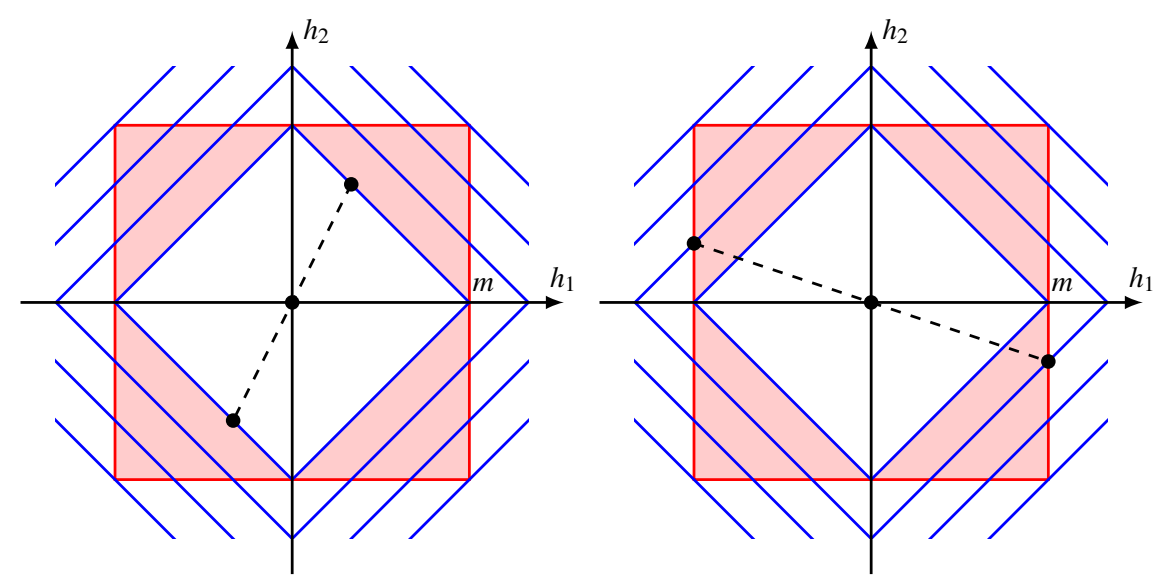

Fig. 1 The trigonometric degree iso-lines (1-norm: diamond shaped iso-lines) versus the product trigonometric degree iso-lines ( $\infty$-norm: iso-lines parallel to the axes). Note that, in contrast to what this 2-dimensional figures suggest, the difference in volume for the enclosing product degree shape increases exponentially with the dimension. Left view: dual lattice points $\boldsymbol{h} \neq \mathbf{0}$ on the 1-norm isoline of distance $m$, i.e., $\|\boldsymbol{h}\|_{1}=m$; the picture shows an enhanced trigonometric degree of $m$, i.e., a trigonometric degree of $m-1$. Right view: no dual lattice points $\boldsymbol{h} \neq \mathbf{0}$ with $\|\boldsymbol{h}\|_{1} \leq m$, i.e., having trigonometric degree at least $m$; in the picture the enhanced trigonometric degree is $m+1$ and thus the trigonometric degree is $m$.

For ease of presentation one often uses the concept of the enhanced trigonometric degree [6] which is defined as the trigonometric degree plus one. In other words, the enhanced trigonometric degree is the distance of the closest non-zero point to the origin of the dual lattice measured in the 1-norm.

We can rewrite kernel (8), getting rid of the sum, using

$$
1+2 \sum_{h=1}^{m} p^{h} \cos (2 \pi h t)=\frac{1-p^{2}-2 p^{m+1} \cos (2 \pi(m+1) t)+2 p^{m+2} \cos (2 \pi m t)}{1+p^{2}-2 p \cos (2 \pi t)}
$$


which can be obtained by tedious calculations starting from the exponential form or using easy manipulations starting from [14, 1.353/3]. However, care must be taken to evaluate this function (in whatever form), especially as $p$ will be chosen small. In Figure 2 one can see what the one-dimensional kernel looks like. (A similar remark is also in place for the kernel used in [13] which has $m=\infty$.)

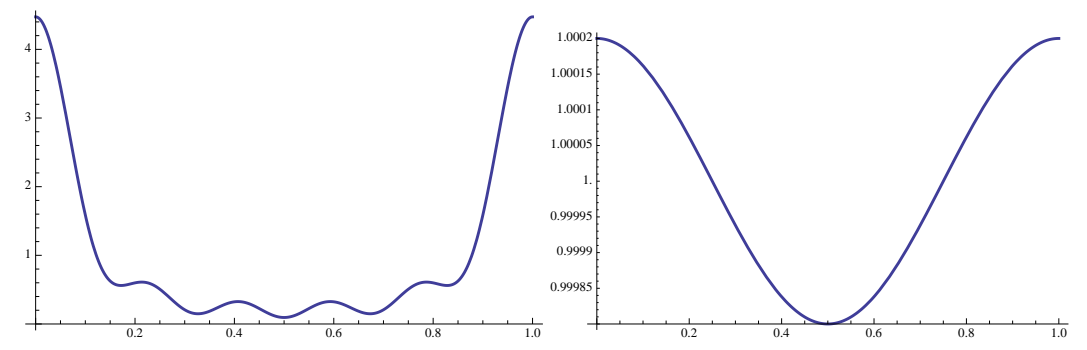

Fig. 2 The one-dimensional kernel $K_{m, p}^{\prime}(x, 0)$, see (8), of the finite dimensional product space which weights Fourier coefficients by the 1-norm. Left: the kernel for $p=2 / 3$ and $m=5$. Right: the kernel for $p=10^{-4}$ and $m=5$.

\section{Distinguishing dual lattice points}

Kernel (8) can be analyzed for the trigonometric degree by looking at the different cases where the squared worst-case error for kernel $K_{m, p}^{\prime}$ is non-zero. We analyze the cases under the premise that the rule has trigonometric degree $m$.

First assume the rule really has trigonometric degree at least $m$, i.e., $\|\boldsymbol{h}\|_{1}>m$ for all dual lattice points $\boldsymbol{h} \neq \mathbf{0}$, and also has dual lattice points for which $\|\boldsymbol{h}\|_{\infty} \leq m$, i.e., dual lattice points in the shaded area of Figure 1. The first 1-norm iso-line on which these points could fall is the one where the 1-norm equals $m+1$ (right image). All points on this iso-line account for a weight of $p^{m+1}$ in the squared worst-case error. Naturally, if there are $1 / p$ dual lattice points on this line, then the squared worst-case error will be at least $1 / p \times p^{m+1}=p^{m}$, which is the weight of the iso-line $\|\boldsymbol{h}\|_{1}=m$.

Conversely, assume the rule actually has degree less than $m$. If there are no dual lattice points on the $m$ th iso-line then the worst-case error is at least $p^{m-1}$. On the other hand, if there would be any dual lattice points on the $m$ th iso-line then the squared worst-case error would as well have a value of order $p^{m}$. Further, note that if there is one dual lattice point at distance $m$, then there is a second one as well as trivially $\|\boldsymbol{h}\|_{1}=\|-\boldsymbol{h}\|_{1}$, thus the squared worst-case error would at least be $2 p^{m}$. So, in the case above, where we have trigonometric degree at least $m$, we would need at least $2 / p$ dual lattice points on the iso-line of weight $m+1$ to have a squared worst-case error of at least $2 p^{m}$.

Unsurprisingly, this shows that the contribution of the dual lattice points with $\|\boldsymbol{h}\|_{1}>m$ and $\|\boldsymbol{h}\|_{\infty} \leq m$ could raise above the level of the dual lattice points with 
$\mathbf{0} \neq\|\boldsymbol{h}\|_{1} \leq m$. This problem can be avoided by choosing $p$ small enough since there is a maximum of integer points which can fall inside the shaded region in Figure 1. A naive but straightforward way is by weighting all points in $\left\{\boldsymbol{h} \in \mathbb{Z}^{d}:\|\boldsymbol{h}\|_{1}>\right.$ $m$ and $\left.\|\boldsymbol{h}\|_{\infty} \leq m\right\}$ by the same factor $p^{m+1}$ which then have a combined weight smaller than two points on the edge of the cross-polytope.

Lemma 1. Given integer $m, d>1$, if one chooses $p$ such that

$$
\frac{1}{p}>2^{d-1}\left((m+1)^{d}-\frac{(d+1) \cdots(d+m)}{m !}\right)
$$

then

$$
\sum_{\substack{\boldsymbol{h} \in \mathbb{Z}^{d} \\\|\boldsymbol{h}\|_{1}>m \\\|\boldsymbol{h}\|_{\infty} \leq m}} p^{m+1}<2 p^{m} .
$$

Proof. We will count the integer points by subtracting the points with $\|\boldsymbol{h}\|_{1} \leq m$ from the points with $\|\boldsymbol{h}\|_{\infty} \leq m$. As we are counting all integer points (instead of only the dual lattice points) we can simplify the count to $\boldsymbol{h}$ with non-negative coordinates and then multiply by a factor of $2^{d}$. Doing so we count all points on the interface between adjacent hypercubes twice, so this is just an approximation.

The number of integer points in $[0, m]^{d}$ is trivially $(m+1)^{d}$. To find the number of integer points in the simplex with vertices $(0,0, \ldots, 0),(m, 0, \ldots, 0),(0, m, \ldots, 0)$, $\ldots,(0,0, \ldots, m)$ we can use the theory of Ehrhart polynomials, see, e.g., [12], from which we find the generating function

$$
\sum_{m \geq 0} a_{m} x^{m}=\frac{1}{(1-x)^{d+1}} .
$$

The $m$ th Maclaurin coefficient is given by

$$
\frac{(d+1) \cdots(d+m)}{m !},
$$

which is the number of integer points inside the simplex. It follows that

$$
\#\left\{\boldsymbol{h} \in \mathbb{Z}^{d}:\|\boldsymbol{h}\|_{1}>m \text { and }\|\boldsymbol{h}\|_{\infty} \leq m\right\} \leq 2^{d}\left((m+1)^{d}-\frac{(d+1) \cdots(d+m)}{m !}\right),
$$

which is sharp for $d=2$. We now want

$$
2^{d}\left((m+1)^{d}-\frac{(d+1) \cdots(d+m)}{m !}\right) p^{m+1}<2 p^{m},
$$

from which the stated result follows.

Given such a choice of $p$ we show that the squared worst-case error in the RKHS with kernel $K_{m, p}^{\prime}$ gives us information on the trigonometric degree. 
Lemma 2. Given an $N$-point rank-1 lattice rule $Q(f ; z, N)$ with generating vector $z$ then for integer $m>1$ and $0<p<1$ chosen as in Lemma 1 we have

0. $e^{2}\left(z, N ; K_{m, p}^{\prime}\right)=0$ if $Q$ has (product) trigonometric degree at least $m$;

and if $e^{2}\left(\boldsymbol{z}, N ; K_{m, p}^{\prime}\right) \neq 0$

1. $\left\lfloor\log _{p} \frac{e^{2}\left(z, N ; K_{m, p}^{\prime}\right)}{2}\right\rfloor \leq m$ if $Q$ has trigonometric degree less than $m$;

2. $\left\lfloor\log _{p} \frac{e^{2}\left(z, N ; K_{m, p}^{\prime}\right)}{2}\right\rfloor>m$ if $Q$ has trigonometric degree at least $m$.

Proof. The case of $e^{2}\left(z, N ; K_{m, p}^{\prime}\right)=0$ is trivial.

Now assume there are no non-zero dual lattice points for which $\|\boldsymbol{h}\|_{1} \leq m$ then

$$
e^{2}\left(\boldsymbol{z}, N ; K_{m, p}^{\prime}\right)=\sum_{\substack{\boldsymbol{h} \cdot \boldsymbol{z} \equiv 0 \\\|\boldsymbol{h}\|_{1}>m \\\|\boldsymbol{h}\|_{\infty} \leq m}} p^{\|\boldsymbol{h}\|_{1}} \leq \sum_{\substack{\boldsymbol{h} \in \mathbb{Z}^{d} \\\|\boldsymbol{h}\|_{1}>m \\\|\boldsymbol{h}\|_{\infty} \leq m}} p^{m+1}<2 p^{m}
$$

due to Lemma 1.

On the other hand if there are non-zero dual lattice points with $\|\boldsymbol{h}\|_{1} \leq m$ then

$$
\begin{aligned}
e^{2}\left(\boldsymbol{z}, N ; K_{m, p}^{\prime}\right) & =\sum_{\substack{\boldsymbol{h} \cdot \boldsymbol{z} \equiv 0 \\
0<\|\boldsymbol{h}\|_{1} \leq m}} p^{\|\boldsymbol{h}\|_{1}}+\sum_{\substack{\boldsymbol{m} \cdot \boldsymbol{z} \equiv 0) \\
\|\boldsymbol{h}\|_{1}>m \\
\|\boldsymbol{h}\|_{\infty} \leq m}} p^{\|\boldsymbol{h}\|_{1}} \\
& \geq \sum_{\substack{\boldsymbol{h} \cdot \boldsymbol{z} \equiv 0 \\
0<\|\boldsymbol{h}\|_{1} \leq m}} p^{\|\boldsymbol{h}\|_{1}} \\
& \geq 2 p^{m} .
\end{aligned}
$$

From these the result follows.

Note that generally it will not be possible to check what the trigonometric degree is when it is larger than $m$. There is always the possibility of a $\boldsymbol{h} \in \Lambda^{\perp}$ such that $\|\boldsymbol{h}\|_{1}=m+\ell$ but $\|\boldsymbol{h}\|_{\infty}>m$ for some $0<\ell<m$ : a dual lattice point outside of $[-m, m]^{d}$ but on a 1-norm iso-line through this hypercube. However, by modifying the choice of $p$ we can determine what the trigonometric degree is when it is smaller than $m$, as stated in the following corollary.

Corollary 1. Given an $N$-point rank-1 lattice rule $Q$ with generating vector $\boldsymbol{z}$ then for integer $m>1$ and $0<p<1$ chosen as

$$
\frac{1}{p}>2^{d-1}\left((m+1)^{d}-(d+1)\right)
$$

we have the additional property that 


$$
\left\lfloor\log _{p} \frac{e^{2}\left(z, N ; K_{m, p}^{\prime}\right)}{2}\right\rfloor=m-\ell+1
$$

if $Q$ has trigonometric degree $m-\ell$ where $0<\ell<m$.

Proof. Following the same reasoning as in the proof of Lemma 1, we find that for $\ell>0$

$\#\left\{\boldsymbol{h} \in \mathbb{Z}^{d}:\|\boldsymbol{h}\|_{1}>m-\ell\right.$ and $\left.\|\boldsymbol{h}\|_{\infty} \leq m\right\} \leq 2^{d}\left((m+1)^{d}-\frac{(d+1) \cdots(d+m-\ell)}{(m-\ell) !}\right)$.

We now want for all possible $0<\ell<m$

$$
2^{d}\left((m+1)^{d}-\frac{(d+1) \cdots(d+m-\ell)}{(m-\ell) !}\right) p^{m+1-\ell}<2 p^{m-\ell},
$$

from which the stated condition on $p$ follows.

Using this condition, suppose the trigonometric degree is $m-\ell$ for some $0<\ell<$ $m$. Then we find

$$
e^{2}\left(\boldsymbol{z}, N ; K_{m, p}^{\prime}\right)=\sum_{\substack{\boldsymbol{h} \cdot \boldsymbol{z} \equiv 0 \\\|\boldsymbol{h}\|_{1}>m-\ell \\\|\boldsymbol{h}\|_{\infty} \leq m}} p^{\|\boldsymbol{h}\|_{1}} \leq \sum_{\substack{\boldsymbol{h} \in \mathbb{Z}^{d} \\\|\boldsymbol{h}\|_{1}>m-\ell \\\|\boldsymbol{h}\|_{\infty} \leq m}} p^{m-\ell+1}<2 p^{m-\ell}
$$

and

$$
e^{2}\left(\boldsymbol{z}, N ; K_{m, p}^{\prime}\right) \geq \sum_{\substack{\boldsymbol{h} \cdot \boldsymbol{z} \equiv 0 \\\|\boldsymbol{h}\|_{1}=m-\ell+1 \\\|\boldsymbol{h}\|_{\infty} \leq m}} p^{\|\boldsymbol{h}\|_{1}} \geq 2 p^{m-\ell+1}
$$

Above we have always lumped together the points to get weighted all by the same weight of $p^{m+1}$. A more careful analysis is possible if we weight each $\boldsymbol{h}$ exactly. This is possible, but we were unable to get such a nice expression as in Lemma 1. The following result could however be used in an algorithmic way to find a $p$ greater than or equal to the one obtained by Lemma 1 .

Lemma 3. Given integer $m, d>1$, if one chooses $p$ such that

$$
\sum_{s=1}^{d} 2^{s}\left(\begin{array}{l}
d \\
s
\end{array}\right)\left(\left(\frac{p^{m+1}-p}{p-1}\right)^{s}-\sum_{k=1}^{m} p^{k}\left(\begin{array}{l}
k-1 \\
s-1
\end{array}\right)\right)<2 p^{m}
$$

then

$$
\sum_{\substack{\boldsymbol{h} \in \mathbb{Z}^{d} \\\|\boldsymbol{h}\|_{1}>m \\\|\boldsymbol{h}\|_{\infty} \leq m}} p^{\|\boldsymbol{h}\|_{1}}<2 p^{m}
$$


Proof. In similar spirit as the previous results we need

$$
\sum_{\substack{\boldsymbol{h} \in \mathbb{Z}^{d} \\\|\boldsymbol{h}\|_{\infty} \leq m}} p^{\|\boldsymbol{h}\|_{1}}-\sum_{\substack{\boldsymbol{h} \in \mathbb{Z}^{d} \\\|\boldsymbol{h}\|_{1} \leq m}} p^{\|\boldsymbol{h}\|_{1}}<2 p^{m}
$$

The weighted integer points inside the hypercube are easy to express as all sums are independent:

$$
\sum_{\substack{h \in \mathbb{Z}^{d} \\\|\boldsymbol{h}\|_{\infty} \leq m}} p^{\|\boldsymbol{h}\|_{1}}=\left(\sum_{h=-m}^{m} p^{|h|}\right)^{d}=\left(1+2 \sum_{h=1}^{m} p^{h}\right)^{d}=\left(1+2 \frac{p^{m+1}-p}{p-1}\right)^{d} .
$$

This can also be written as

$$
\left(1+2 \frac{p^{m+1}-p}{p-1}\right)^{d}=\sum_{s=0}^{d}\left(\begin{array}{l}
d \\
s
\end{array}\right)\left(2 \frac{p^{m+1}-p}{p-1}\right)^{s}=1+\sum_{s=1}^{d} 2^{s}\left(\begin{array}{l}
d \\
s
\end{array}\right)\left(\frac{p^{m+1}-p}{p-1}\right)^{s} .
$$

For the weighted points inside the cross-polytope we have the number of points at distance $k$ to be

$$
\sum_{\substack{\boldsymbol{h} \in \mathbb{Z}^{d} \\
\|\boldsymbol{h}\|_{1}=k}} 1=\sum_{\substack{\boldsymbol{h} \in \mathbb{Z}^{d} \\
\|\boldsymbol{h}\|_{1} \leq k}} 1-\sum_{\substack{\boldsymbol{h} \in \mathbb{Z}^{d} \\
\|\boldsymbol{h}\|_{1} \leq k-1}} 1=\sum_{s \geq 0} 2^{s}\left(\begin{array}{l}
d \\
s
\end{array}\right)\left(\left(\begin{array}{l}
k \\
s
\end{array}\right)-\left(\begin{array}{c}
k-1 \\
s
\end{array}\right)\right)=\sum_{s \geq 1} 2^{s}\left(\begin{array}{l}
d \\
s
\end{array}\right)\left(\begin{array}{c}
k-1 \\
s-1
\end{array}\right)
$$

for $k \geq 1$, cf. (6). As such, the weighted integer points inside the cross-polytope are given by

$$
1+\sum_{k=1}^{m} p^{k} \sum_{s \geq 1} 2^{s}\left(\begin{array}{l}
d \\
s
\end{array}\right)\left(\begin{array}{l}
k-1 \\
s-1
\end{array}\right)=1+\sum_{s=1}^{d} 2^{s}\left(\begin{array}{l}
d \\
s
\end{array}\right)\left(\sum_{k=1}^{m} p^{k}\left(\begin{array}{l}
k-1 \\
s-1
\end{array}\right)\right) .
$$

(Depending on the choice of $d$ and $m$ the sum over $k$ might vanish partly or even completely because of the properties of the binomial coefficient, cf. (6).) From here the result follows.

\section{A modification of the $\mathrm{CKN}$ weighted-degree algorithm}

The algorithm in [5] is a component-by-component algorithm, see, e.g., [25]. This means that one constructs the generating vector $z$ one component at a time, first generating a one dimensional vector, then a two dimensional, etc, always keeping the previous choices fixed.

Using the results from the previous section, we can modify the algorithm from [5] as follows. Starting from a $d$-dimensional generating vector with trigonometric degree $m_{d}$, we "guess" (as explained below) the trigonometric degree $\tilde{m}_{d+1}$ that can 
be achieved in $d+1$ dimensions. We then use kernel $K_{\tilde{m}_{d+1}, p}^{\prime}$, given in (8), with an appropriate choice for $p$, e.g., given by Lemma 1, to calculate the squared worst-case error for each possible choice of $z_{d+1}$. For this we consider all $z \in \mathbb{Z}_{N}^{\times}$(where $\mathbb{Z}_{N}^{\times}$are all positive integers relatively prime to $N$ and smaller than $N$, i.e., the multiplicative group modulo $N$ ). This step might possibly be repeated for different choices of $\tilde{m}_{d+1}$ if our initial guess turned out to be incorrect, making use of Lemma 2. As we have chosen a tensor product form kernel, the calculation of the worst-case error for all possible choices $z \in \mathbb{Z}_{N}^{\times}$can be done using Fast Fourier Transformations (FFTs) using the techniques from $[20,22,21]$ in time $O(N \log N)$ for each guess of $\tilde{m}_{d+1}$. The final trigonometric degree that we settle on will be denoted by $m_{d+1}$.

As in [5] we try to achieve a good trigonometric degree and at the same time obtain an almost optimal worst-case error in a Korobov space. For this we also calculate the worst-case error using kernel $K_{\alpha}$, see (4), and find the $z \in \mathbb{Z}_{N}^{\times}$which minimizes this worst-case error and at the same time achieves the trigonometric degree $m_{d+1}$ (found by the calculations based on $K_{m_{d+1}, p}^{\prime}$ ). The final choice of $z$ is then fixed as $z_{d+1}$. The calculation of the worst-case error in the Korobov space might also be done using FFTs in time $O(N \log N)$.

When the number of points is sufficiently large, then [5, Theorem 3] shows that such lattice rules exist and can be found in a component by component way. For completeness we repeat that result here (which is stated for a prime number of points due to technicalities), slightly adjusted to the context of the (unweighted) trigonometric degree here. (The subsequent theorem also uses "product weights" $\gamma_{j}$ to build a weighted function space. Such weighted spaces are a standard tool in tractability analysis but are of no real concern in this paper and can be safely ignored. Further information can be found in, e.g., [26].)

Theorem 1 (From [5, Theorem 3]). Let $c>1$ be fixed, $m$ be given, and let $N$ be a prime number satisfying

$$
N>\max \left(m, 1+\frac{c}{c-1} \frac{\left|\mathscr{A}_{d+1}(m)\right|-\left|\mathscr{A}_{d}(m)\right|-2 m}{2}\right) .
$$

Suppose we already have a $z \in\left(\mathbb{Z}_{N}^{\times}\right)^{d}$ for which

$$
e^{2}\left(z, N ; K_{m}\right)=0
$$

i.e., the rule has trigonometric degree at least $m$, and

$$
e^{2}\left(z, N ; K_{\alpha}\right) \leq\left(\frac{c}{N-1} \prod_{j=1}^{d}\left(1+2 \gamma_{j}^{\lambda} \zeta(\alpha \lambda)\right)\right)^{1 / \lambda} \quad \text { for all } \lambda \in(1 / \alpha, 1],
$$

i.e., the rule has near optimal worst-case error in the Korobov space with smoothness $\alpha$. Then there is "at least one" $z_{d+1} \in \mathbb{Z}_{N}^{\times}$such that we achieve trigonometric degree $m$

$$
e^{2}\left(\left(z, z_{d+1}\right), N ; K_{m}\right)=0,
$$


and near optimal worst-case error

$$
e^{2}\left(\left(z, z_{d+1}\right), N ; K_{\alpha}\right) \leq\left(\frac{c}{N-1} \prod_{j=1}^{d+1}\left(1+2 \gamma_{j}^{\lambda} \zeta(\alpha \lambda)\right)\right)^{1 / \lambda} \quad \text { for all } \lambda \in(1 / \alpha, 1] .
$$

This choice of $N$ however was argued to be much higher than necessary, so in the practical implementation the condition was omitted. Here we will follow the same argument: we omit the condition on $N$ and try to achieve the highest possible trigonometric degree possible. From [11] we note the known minimum number of points needed to achieve a prescribed trigonometric degree $m$ in $d$ dimensions:

$$
N_{\min }(m, d) \geq\left|\mathscr{A}_{d}\left(\left\lfloor\frac{m}{2}\right\rfloor\right)\right|= \begin{cases}O\left(m^{d}\right), & \text { if } d \leq m, \\ O\left((2 d)^{m / 2}\right), & \text { if } m \leq d .\end{cases}
$$

More specifically, the attainable lower bound in 2 dimensions, again, see [11], is given by

$$
N_{\min }(m, 2)= \begin{cases}2 k^{2}+2 k+1, & \text { for } m=2 k \\ 2 k^{2}+4 k+2, & \text { for } m=2 k+1\end{cases}
$$

This brings us back to the "guessing" of the trigonometric degree. First note that the range of possible trigonometric degrees is quite limited. As an estimate in two dimensions we could use $m<\sqrt{2 N}$. It follows that for a fixed $N$ and increasing $d$ (as in a component-by-component algorithm) the achievable trigonometric degree will decrease exponentially, see (9). This enables us to guess the trigonometric degree rather easily. To start off the process we use that the trigonometric degree in the first dimension always equals $N-1$ (under the condition that $z_{1}$ is relatively prime to $N$ ), for the second dimension we can start from the explicit lower bound, i.e., guess $m<\sqrt{2 N}$, and from then on we can assume exponential decrease. Moreover, if we never underestimate $m$, then by choosing $p$ as in Corollary 1 we can determine the trigonometric degree from the squared worst-case error. Summarizing, we have the following algorithm:

Algorithm 1 For given $d_{\max }$, wanted degree $\hat{m}_{d_{\max }} \geq 1$ in $d_{\max }$ dimensions, $\alpha>1$ and choosing $N \geq\left|\mathscr{A}_{d_{\max }}\left(\left\lfloor\hat{m}_{d_{\max }} / 2\right\rfloor\right)\right|$, then:

1. Set $z_{1}=1$.

2. For each $d=1, \ldots, d_{\max }-1$ with $z=\left(z_{1}, \ldots, z_{d}\right)$ already fixed do the following:

a. Guess the trigonometric degree $m_{d+1}$ (preferably do not underestimate), and choose a $p$ small enough, e.g., as in Lemma 1 or Corollary 1.

b. For each possible component $z \in \mathbb{Z}_{N}^{\times}$, calculate $e^{2}\left(\left(z, z_{d+1}\right), N ; K_{m_{d+1}, p}^{\prime}\right)$.

If there is no choice with trigonometric degree $m_{d+1}$ then guess again and repeat this step, otherwise set the trigonometric degree $m_{d+1}$.

c. Set $z_{d+1}$ to be the $z \in \mathbb{Z}_{N}^{\times}$that minimizes $e^{2}\left(\left(z, z_{d+1}\right), N ; K_{\alpha}\right)$ and has degree $m_{d+1}$. 
Corollary 2. Given Algorithm 1, we have that the complexity of construction up to $d$ dimensions is $O\left(d N(\log N)^{2}\right)$.

Proof. Assuming we need to repeat the guess $T$ times, the cost per iteration is $O(T N \log N)$. As the relation between the number of points $N$ and the achievable trigonometric degree is exponential for increasing $d$, see (9), we can assume $T=O(\log N)$ worst case. Consequently the $\operatorname{cost}$ is $O\left(N(\log N)^{2}\right)$ and the complexity of construction up to $d$ dimensions is $O\left(d N(\log N)^{2}\right)$.

We remark that the construction cost given assumes unit cost for all basic arithmetic operations on the computing device. Based on the smallness of $p$ this will almost always mean arbitrary precision calculations for which this assumption is not quite correct (depending on the needed precision the deviation will become larger). An analysis of the practical implications for an actual implementation of this algorithm is therefore left for future research; but we make some developments in this area in the remainder of the paper. (We note that the full study of this would imply a numerical analysis of the computation of the worst-case error. As far as we know, this has not been studied yet.) To give an example of the technical complications: if the needed precision is very high, then it will become necessary to use FFTs which minimize the number of multiplications; or to use other algorithms to execute the underlying circular convolution.

We remark as well that in each iteration of the algorithm, we are in fact more or less computing the shortest vector in circa $N \approx\left|\mathbb{Z}_{N}^{\times}\right|$dual lattices. So somewhere we expect to get bitten by the exponential complexity in $d$ of the general problem of shortest vector computations. In that respect, the proposed algorithm looks quite good and it seems we can reduce the complexity by exploiting the specifics of our problem.

\section{An improvement on $p$}

It is clear that our choice of $p$ is far too conservative; it was a very crude underestimate based on a worst case argument. We simulated $10^{3}$ random numbers $N$ between 100 and 4001, together with 5-dimensional integer vectors $z$ with elements between 1 and $N$. Then, for each dimension between 2 and 5 we calculated the enhanced trigonometric degree explicitly (which we denote in this section by $m$ for ease of notation), after which we checked for all $\boldsymbol{h}$ in $\left\{\boldsymbol{h}:\|\boldsymbol{h}\|_{\infty} \leq m\right.$ and $\left.\|\boldsymbol{h}\|_{1}>m\right\}$ whether they satisfy $\boldsymbol{h} \cdot \boldsymbol{z} \equiv 0(\bmod N)$. This gave us $10^{3}$ trigonometric degrees and the corresponding number of dual lattice points in $\Lambda_{m}^{\perp}:=\left\{\boldsymbol{h}: \boldsymbol{h} \in \Lambda^{\perp},\|\boldsymbol{h}\|_{\infty} \leq\right.$ $m$ and $\left.\|\boldsymbol{h}\|_{1}>m\right\}$ for each dimension. In Table 1, the maximum $\left|\Lambda_{m}^{\perp}\right|$ that was found for each trigonometric degree encountered is reported. We also calculated the theoretical bound

$$
\Omega_{m}=2^{d}\left((m+1)^{d}-\frac{(d+1) \cdots(d+m)}{m !}\right)
$$




\begin{tabular}{l||cc|cc|cc|cc}
\multicolumn{1}{c|}{} & $d=2$ & & $d=3$ & & $d=4$ & & $d=5$ & \\
$m$ & $\left|\Lambda_{m}^{\perp}\right|$ & $\Omega_{m}$ & $\left|\Lambda_{m}^{\perp}\right|$ & $\Omega_{m}$ & $\left|\Lambda_{m}^{\perp}\right|$ & $\Omega_{m}$ & $\left|\Lambda_{m}^{\perp}\right|$ & $\Omega_{m}$ \\
\hline 2 & 2 & 12 & 10 & 136 & 14 & 1056 & 62 & 7104 \\
3 & 0 & 24 & 8 & 352 & 88 & 3536 & 312 & 30976 \\
4 & 4 & 40 & 20 & 720 & 122 & 8880 & 630 & 95968 \\
5 & 4 & 60 & 22 & 1280 & 136 & 18720 & 1416 & 240768 \\
6 & 4 & 84 & 20 & 2072 & 174 & 35056 & 1350 & 523040 \\
7 & 4 & 112 & 32 & 3136 & 202 & 60256 & 1298 & 1023232 \\
8 & 4 & 144 & 36 & 4512 & 250 & 97056 & 1346 & 1848384 \\
9 & 4 & 180 & 28 & 6240 & 222 & 148560 & 1560 & 3135936 \\
10 & 4 & 220 & 32 & 8360 & 210 & 218240 & 1508 & 5057536 \\
11 & 4 & 264 & 36 & 10912 & 222 & 309936 & 1660 & 7822848 \\
12 & 4 & 312 & 34 & 13936 & 222 & 427856 & & \\
13 & 4 & 364 & 32 & 17472 & 220 & 576576 & & \\
14 & 6 & 420 & 30 & 21560 & 210 & 761040 & & \\
15 & 4 & 480 & 32 & 26240 & & & & \\
16 & 6 & 544 & 32 & 31552 & & & & \\
17 & 4 & 612 & 36 & 37536 & & & & \\
18 & 6 & 684 & 38 & 44232 & & & & \\
19 & 4 & 760 & 34 & 51680 & & & & \\
20 & 4 & 840 & 32 & 59920 & & & & \\
21 & 4 & 924 & 34 & 68992 & & & & \\
22 & 4 & 1012 & 34 & 78936 & & & & \\
23 & 4 & 1104 & 36 & 89792 & & & & \\
24 & 4 & 1200 & 36 & 101600 & & & & \\
25 & 4 & 1300 & 34 & 114400 & & & & \\
26 & 4 & 1404 & & & & & & \\
27 & 4 & 1512 & & & & & & \\
28 & 4 & 1624 & & & & & & \\
29 & 4 & 1740 & & & & & & \\
30 & 4 & 1860 & & & & & & \\
31 & 4 & 1984 & & & & & & \\
32 & 4 & 2112 & & & & & & \\
33 & 4 & 2244 & & & & & & \\
34 & 6 & 2380 & & & & & & \\
35 & 4 & 2520 & & & & & & \\
36 & 6 & 2664 & & & & & & \\
37 & 4 & 2812 & & & & & & \\
38 & 6 & 2964 & & & & & & \\
39 & 4 & 3120 & & & & & & \\
40 & 4 & 3280 & & & & & & \\
& 4 & & & &
\end{tabular}

Table 1 The enhanced trigonometric degrees found in a sample of random generating vectors up to 5 dimensions, together with the corresponding maximum number of dual lattice points $\left|\Lambda_{m}^{\perp}\right|$ found and the theoretical bound $\Omega_{m}$, used for constructing $p$ in Lemma 1.

used in Lemma 1 for constructing $p$ to compare against the numerical experiment. It seems that $\Omega_{m}$ greatly overestimates the possible number of dual lattice points. More specifically, we have the following lemma for the two-dimensional case.

Lemma 4. For $d=2$, the maximum number of points in $\Lambda_{m}^{\perp}$ is bounded by 6 for any $m$.

Proof. This lemma can be proven by noticing that for rank-1 lattice rules all dual lattice points lie equidistantly on equidistant parallel hyperplanes.

This lemma illustrates, at least for $d=2$, that the number of possible dual lattice points is fixed regardless of the trigonometric degree, whereas $\Omega_{m}$, used for calculating $p$ in our algorithm, increases with $m$. From Table 1 there seems to be some 
evidence that the maximum number of points in $\Lambda_{m}^{\perp}$ is much smaller than $\Omega_{m}$ also in higher dimensions.

Lemma 1 has been written in a general sense, without using any information on the actual underlying point set. If we specialize to rank-1 rules we can get a better estimate. We start from the following easy result.

Lemma 5. Given an $N$-point rank-1 lattice rule with generating vector $z \in\left(\mathbb{Z}_{N}^{\times}\right)^{d}$ modulo $N$, with $N$ prime, then there are $N^{d-1}$ dual lattice points modulo $N$ (i.e., in $\left.[0, N)^{d}\right)$.

Proof. An integer point $\boldsymbol{h} \in \mathbb{Z}^{d}$ is part of the dual lattice if

$$
h_{1} z_{1}+h_{2} z_{2}+\cdots+h_{d} z_{d} \equiv 0 \quad(\bmod N) .
$$

Now fix any choice of $h_{j} \in \mathbb{Z}_{N}$ except one, say $h_{1}$, then for $a=\left(h_{2} z_{2}+\cdots+h_{d} z_{d}\right)$ there is a unique solution, since $z_{1} \in \mathbb{Z}_{N}^{\times}$, for $h_{1}$ in

$$
h_{1} z_{1}+a \equiv 0 \quad(\bmod N) .
$$

The same conclusion could be drawn if fixing any other $d-1$ components. As there were $N^{d-1}$ choices for the other $h_{j}$ the dual lattice has $N^{d-1}$ points in $[0, N)^{d}$.

Similarly to the previous lemma we obtain an estimate for the dual lattice points inside $[-m, m]^{d}$.

Corollary 3. Under the same conditions as for Lemma 5 , there are at most $(2 m+$ $1)^{d-1}$ dual lattice points in $[-m, m]^{d}$.

Note that this seems always smaller than $\Omega_{m}$. However, this result is only valid for rank-1 lattice rules, whereas Lemma 1 remains valid for higher rank lattice rules. Therefore, we opted to keep Lemma 1 as a guideline although this estimate will get us a smaller $p$. A practical implementation for rank-1 rules could however make use of Corollary 3.

In closing this section we want to remark that, apart from making $p$ larger by using a more careful analysis, we can also make $p$ larger by sorting out bad cases as we go. E.g., the following lemma shows that as soon as we have fixed a component of $\boldsymbol{z}$, then several multiples modulo $N$ must not be considered again as valid choices in the next dimensions; as such we should not care about the actual $p$ value we would need for these very bad rules.

Lemma 6. Given an $N$-point rank-1 lattice rule with generating vector $z \in \mathbb{Z}_{N}^{d}$ in $d \geq 2$ dimensions, then as soon as there is a repeated component (modulo $N$ ) in $z$, the trigonometric degree is just 1 . Moreover, if one component, say $z_{j}$, is $-t$ times a multiple of another (modulo $\left.N /\left(z_{i}, N\right)\right)$, say $z_{i}$, and $t \not \equiv 0\left(\bmod N /\left(z_{i}, N\right)\right)$, then the trigonometric degree is at most $t$.

Proof. We just prove the most general case. Consider the vector $\boldsymbol{h}$ which is zero everywhere except for the two components where $z_{i} \equiv a(\bmod N)$ and $z_{j} \equiv-t a$ $(\bmod N /(a, N))$. We get the equation 


$$
h_{i} z_{i}+h_{j} z_{j} \equiv 0 \quad(\bmod N)
$$

which, with $(a, N)$ the greatest common divisor of $a$ and $N$, is equivalent to

$$
\frac{a}{(a, N)} h_{i}-\frac{t a}{(a, N)} h_{j} \equiv 0 \quad(\bmod N /(a, n)),
$$

where we have to assert that $t \not \equiv 0(\bmod N /(a, n))$ such that the problem still involves $h_{i}$ and $h_{j}$. Multiplying by the multiplicative inverse of $a /(a, N)$ we obtain

$$
h_{i}-t h_{j} \equiv 0 \quad(\bmod N /(a, n))
$$

which clearly has a non-trivial solution $h_{i}=t$ and $h_{j}=1$. It follows that the enhanced trigonometric degree is at most $\|\boldsymbol{h}\|_{1}=t+1$ and thus the trigonometric degree can be at most $t$.

As a consequence of this last lemma we note that in Algorithm 1 as the algorithm progresses from dimension to dimension and as we have fixed $N$ from the beginning - thus limiting the achievable trigonometric degree - the possible choices for the next $z_{d+1}$ are much less than the elements of $\mathbb{Z}_{N}^{\times}$. We would hope that exploiting this knowledge would enable us to take much larger choices of $p$, as the bad choices will be the ones with the most points close to $\mathbf{0}$.

\section{Conclusion and future work}

We proposed a component-by-component algorithm to construct rules of good trigonometric degree by making use of a finite dimensional, exponentially decaying, reproducing kernel Hilbert space. The analysis of the algorithm has been tackled from an "existence" point of view, that is, we have proven that such an algorithm exist, and even explicitly given the algorithm outline, but we did not consider practical implementation aspects. Working out the technical details of the algorithm is of considerable complexity and left for future work. Some initial results in that direction have been included.

Acknowledgements The authors would like to thank the two anonymous referees for useful comments on the manuscript.

\section{References}

1. M. Beckers and R. Cools. A relation between cubature formulae of trigonometric degree and lattice rules. In H. Brass and G. Hämmerlin, editors, Numerical integration IV (Oberwolfach, 1992), pages 13-24. Birkhäuser Verlag, 1993. 
2. R. Cools. More about cubature formulas and densest lattice packings. East Journal on Approximations, 12(1):37-42, 2006.

3. R. Cools and H. Govaert. Five- and six-dimensional lattice rules generated by structured matrices. J. Complexity, 19(6):715-729, 2003.

4. R. Cools, F. Y. Kuo, and D. Nuyens. Constructing embedded lattice rules for multivariate integration. SIAM J. Sci. Comput., 28(6):2162-2188, 2006.

5. R. Cools, F. Y. Kuo, and D. Nuyens. Constructing lattice rules based on weighted degree of exactness and worst case error. Computing, 87(1-2):63-89, 2010.

6 . R. Cools and J. N. Lyness. Three- and four-dimensional $K$-optimal lattice rules of moderate trigonometric degree. Math. Comp., 70(236):1549-1567, 2001.

7. R. Cools, E. Novak, and K. Ritter. Smolyak's construction of cubature formulas of arbitrary trigonometric degree. Computing, 62(2):147-162, 1999.

8. R. Cools and D. Nuyens. A Belgian view on lattice rules. In A. Keller, S. Heinrich, and H. Niederreiter, editors, Monte Carlo and Quasi-Monte Carlo Methods 2006, pages 3-21. Springer-Verlag, 2008.

9. R. Cools and D. Nuyens. Extensions of Fibonacci lattice rules. In P. L'Écuyer and A. B. Owen, editors, Monte Carlo and Quasi-Monte Carlo Methods 2008, pages 1-12. SpringerVerlag, 2009.

10. R. Cools and A. V. Reztsov. Different quality indexes for lattice rules. J. Complexity, 13(2):235-258, 1997.

11. R. Cools and I. H. Sloan. Minimal cubature formulae of trigonometric degree. Math. Comp., 65(216):1583-1600, 1996.

12. J. A. De Loera, J. Rambau, and F. Santos. Triangulations, volume 25 of Algorithms and Computation in Mathematics. Springer-Verlag, 2010.

13. J. Dick, F. Pillichshammer, G. Larcher, and H. Woźniakowski. Exponential convergence and tractability of multivariate integration for Korobov spaces. Math. Comp., 80(274):905-930, 2011.

14. I. S. Gradshteyn and I. M. Ryzhik. Table of Integrals, Series and Products. Academic Press, 7th edition, 2007.

15. F. J. Hickernell. Lattice rules: How well do they measure up? In P. Hellekalek and G. Larcher, editors, Random and Quasi-Random Point Sets, pages 109-166. Springer-Verlag, Berlin, 1998.

16. J. N. Lyness. Notes on lattice rules. J. Complexity, 19(3):321-331, 2003.

17. J. N. Lyness and T. Sørevik. Four-dimensional lattice rules generated by skew-circulant matrices. Math. Comp., 73(245):279-295, 2004.

18. J. N. Lyness and T. Sørevik. Five-dimensional $K$-optimal lattice rules. Math. Comp., 75(255):1467-1480, 2006.

19. H. Niederreiter. Random Number Generation and Quasi-Monte Carlo Methods. Number 63 in Regional Conference Series in Applied Mathematics. SIAM, 1992.

20. D. Nuyens and R. Cools. Fast algorithms for component-by-component construction of rank-1 lattice rules in shift-invariant reproducing kernel Hilbert spaces. Math. Comp., 75(254):903920, 2006.

21. D. Nuyens and R. Cools. Fast component-by-component construction, a reprise for different kernels. In H. Niederreiter and D. Talay, editors, Monte Carlo and Quasi-Monte Carlo Methods 2004, pages 371-385. Springer-Verlag, 2006.

22. D. Nuyens and R. Cools. Fast component-by-component construction of rank-1 lattice rules with a non-prime number of points. J. Complexity, 22(1):4-28, 2006.

23. N. N. Osipov, R. Cools, and M. V. Noskov. Extremal lattices and the construction of lattice rules. Appl. Math. Comput., 217(9):4397-4407, 2011.

24. I. H. Sloan and S. Joe. Lattice Methods for Multiple Integration. Oxford Science Publications, 1994.

25. I. H. Sloan and A. V. Reztsov. Component-by-component construction of good lattice rules. Math. Comp., 71(237):263-273, 2002.

26. I. H. Sloan and H. Woźniakowski. When are quasi-Monte Carlo algorithms efficient for high dimensional integrals? J. Complexity, 14(1):1-33, 1998. 